\title{
Analysis using higher-order XFEM: implicit representation of geometrical features from a given parametric representation
}

\author{
M. Moumnassi ${ }^{1, a}$, S.P.A. Bordas ${ }^{2, b}$, R. Figueredo ${ }^{1}$ And P. Sansen ${ }^{1}$ \\ 1 ESIEE-Amiens, 14 quai de la Somme, 80082 Amiens Cedex 2, France \\ 2 School of Engineering, Institute of Mechanics and Advanced Materials, Cardiff University, Queen's Buildings, The Parade, \\ Cardiff CF24 3AA, UK
}

Received 21 June 2013, Accepted 3 May 2014

\begin{abstract}
We present a promising approach to reduce the difficulties associated with meshing complex curved domain boundaries for higher-order finite elements. In this work, higher-order XFEM analyses for strong discontinuity in the case of linear elasticity problems are presented. Curved implicit boundaries are approximated inside an unstructured coarse mesh by using parametric information extracted from the parametric representation (the most common in Computer Aided Design CAD). This approximation provides local graded sub-mesh (GSM) inside boundary elements (i.e. an element split by the curved boundary) which will be used for integration purpose. Sample geometries and numerical experiments illustrate the accuracy and robustness of the proposed approach.
\end{abstract}

Key words: Higher-order XFEM / curved boundary / parametric functions / implicit boundary representation / graded sub-mesh (GSM)

\section{Introduction}

High-order finite element methods offer high accuracy and rates of convergence using coarse meshes. However, applying higher-order finite elements to curved domains requires (i) the need to conform curved mesh entities to curved boundaries and (ii) a correct treatment for higherorder integration rules to compute volume and boundary integrals. Moreover, the construction of curved element meshes may lead to invalid curved elements near a curved boundary, for example due to an excessive distortion. Therefore, it is necessary to develop efficient procedures to detect the validity of mesh elements and to correct the invalid elements ensuring that the Jacobian determinant is strictly positive.

Our interests in simplification of meshes, correct treatment of numerical integration over elements with curved boundaries, motivated us to seek a flexible and simple technique, while retaining benefits of the high-order finite element method (FEM). Ideally, the information about the domain geometry should be independent of the finite element mesh size or its order of interpolation. A large number of researchers [1-7] have investigated a variety of

\footnotetext{
a Corresponding author: mohammed.moumnassi@ymail.com

b stephane.bordas@alum.northwestern.edu
}

concepts which do not require the generation of a conforming mesh and modelling geometrical features independently of the finite element mesh used for analysis. These concepts differ from each other on the following points:

Types of numerical methods: eXtended finite element method (XFEM) [8], the generalized finite element method (GFEM) [9], finite cell method (FCM) [2,10], Cartesian Grid FEM (cg-FEM) [7], etc. Types of the background mesh grid: structured $[2,4]$ or unstructured [1] coarse mesh.

Techniques to represent boundaries: explicit surface representations [5], level set representation $[1,4]$, parametric function to level set/signed distance representation $[1,11]$, medical image modalities $[2,4]$.

Strategies to construct boundaries over the background mesh grid: Quadtree/Octree partition of space $[2,4]$, degenerated and graded sub-meshes (DSM and GSM) in 2D/3D [1].

Here, we use a background unstructured simplex mesh that serves to construct the computational domain and serves for analysis by higher-order shape functions. For this purpose, we use the implicit representation (Level Set Description) to define the geometrical features to 


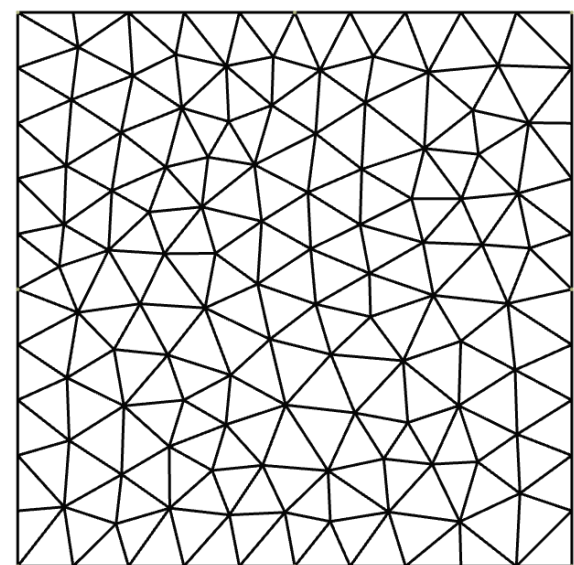

(a)

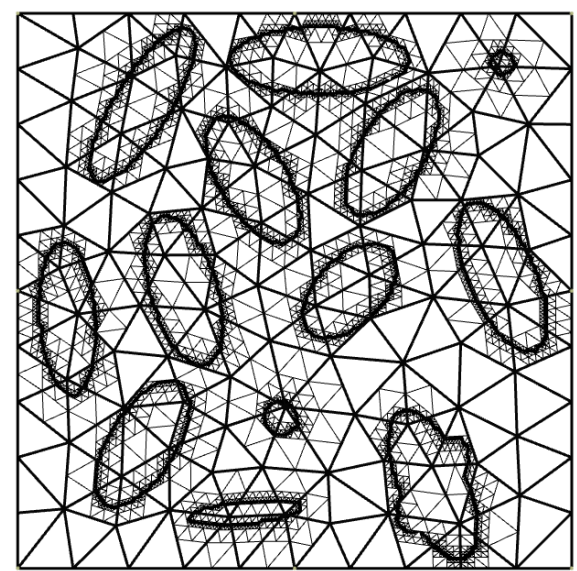

(b)

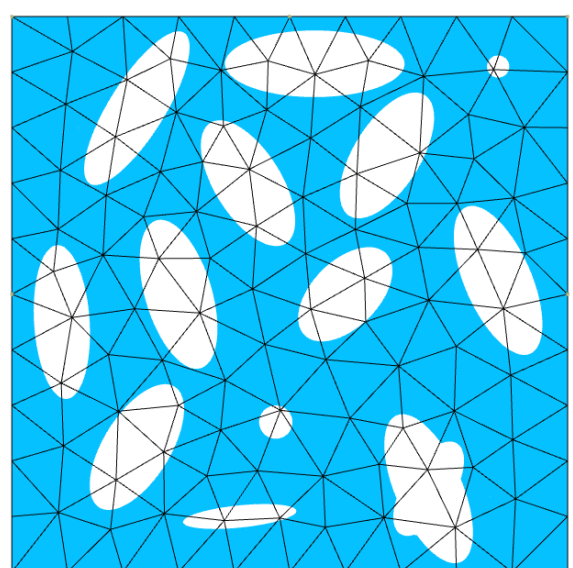

(c)

Fig. 1. Microstructure containing a distribution of voids with different sizes and shapes. (a) Unstructured coarse mesh for finite element analysis. (b) Adaptive sub-mesh refinement of level $(n=7)$ using GSM. (c) Implicit computational domain.

represent domain boundaries and XFEM for analysis. To construct the curved boundaries with minimal dependence on this background mesh, we use the hybrid method proposed by Moumnassi et al. [1] which exploits the advantages of the parametric and implicit (level set) representations. We employ a graded sub-mesh (GSM) [1] strategy to construct curved domain boundaries over the background mesh, and for the integration of the weak form. The proposed representation guarantees the desired approximation a priori of the original object and also provides an efficient numerical integration where integrals over curved domains and curved boundaries are based on the standard Gauss quadrature.

Our approach shares some similarities with the Finite Cell Method [2] and the recent one proposed by Legrain et al. [4] in which use high-order XFEM. However, it can be seen as more general in that it is possible to construct the approximation directly from an arbitrary parametric definition of the object (the most common in Computer Aided Design CAD) and handles corners and sharp edges exactly. Moreover, an arbitrary background mesh (unstructured) can be used.

\section{Implicit curved domain based on parametric representation}

Recently, Moumnassi et al. [1] developed a hybrid parametric/implicit representation well-suited to methods based on fixed grids such as the extended finite element method (XFEM). They showed that it was possible, using a marching algorithm for automatic conversion from a parametric surface into a zero level set defined on a narrow band of the background mesh, to construct a finer graded sub-mesh (GSM) inside the split elements, to build an implicit computational domain independently of the finite element mesh size or its order of interpolation. A framework based on multiple level sets, constructive solid geometry (CSG) and a cutting method was used to construct a fully implicit domain for analysis. This framework will be considered in this work to construct curved boundaries from parametric functions and to build implicit computational domains independently of the background finite element mesh size that will be used for XFEM analysis.

Figure 1 shows an example to construct an implicit computational domain independently of the background finite element mesh size. Geometrical features describing curved boundaries are based on parametric functions which are converted into multiple zero level sets on the background mesh grid. The marching algorithm locates the narrow band that encloses the curved boundary from all elements in the mesh, in which only the selected elements will be used to construct the graded sub-mesh (GSM). The parametric information is used as a guide to generate the profile of the curved region inside the finer graded mesh and the level set resulted from this conversion is used to classify the sub-elements into the solid part and the void part. This sub-mesh is only used to carefully locate the curved regions inside the set of mesh elements which contains the zero level set and to generate Gauss points to integrate the weak form, which differentiates them from finite elements.

Now we have the necessary tools for analysis: computational domain and the background coarse mesh that will serve as support for shape functions. The next step will be devoted to adapting the use of XFEM for our approach.

\section{Finite element analysis}

We consider a background mesh grid $G r$ (see Fig. 2a) that serves as support for the finite element shape functions of order $p$. Gr encloses a computational domain $\Omega_{h} \subset \mathbb{R}^{n},(n=2,3)$ and its boundaries $\Gamma_{h}$. The computational domain divides $G r$ into three sets: the sets of 


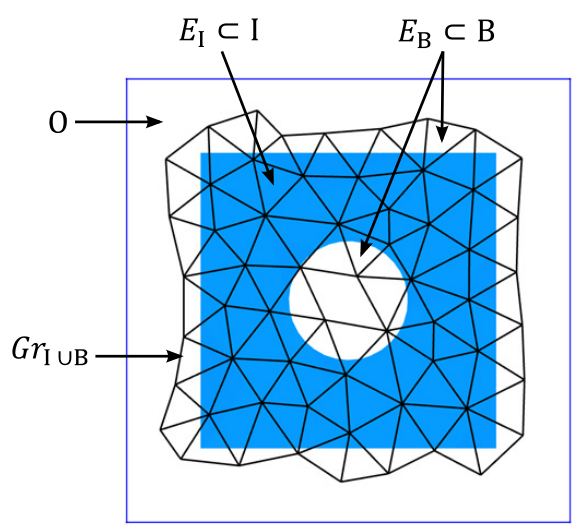

(a)

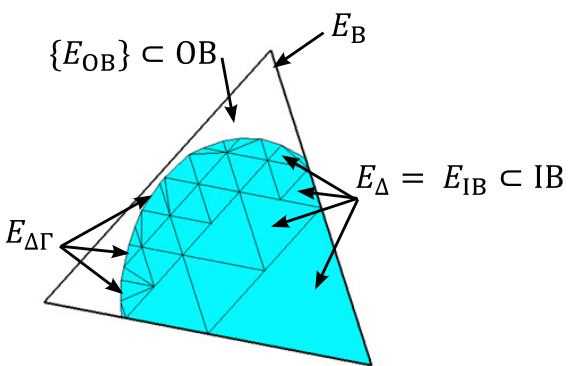

(b)

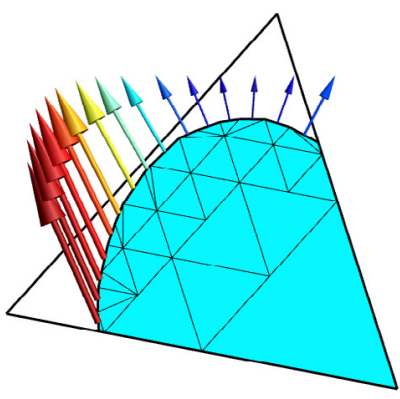

(c)

Fig. 2. (a) Sets of elements $I, B, O$ and $G r_{I \cup B}$ inside an unstructured mesh grid. (b) Sub-elements $E_{\Delta}$ and $E_{\Delta_{\Gamma}}$ resulting from a graded sub-mesh refinement of level $(n=3)$. (c) Example of boundary integrals on $E_{\Delta_{\Gamma}}$.

elements $I$ (Interior), $B$ (Boundary) and $O$ (Outside). Interior elements $E_{I}$ are those which are completely inside $\Omega_{h}$; exterior elements $E_{O}$ which are completely outside $\Omega_{h}$; boundary elements $E_{B}$ which are split by $\Gamma_{h}$. The union of the two sets of elements $I$ and $B$, denoted $G r_{I \cup B}$ covers entirely the computational domain. In the case of modelling void-material interfaces by XFEM, the spatial discretization of PDEs is done on $G r_{I \cup B}$, and the degrees of freedom on the set of elements $O$ will be deleted from the weak formulation.

\subsection{Numerical integration}

The set of elements $B$ which cover boundaries of the computational domain, in turn, is divided into two subsets of elements (see Fig. 2b): $I B$ (inside the boundary $\Gamma_{h}$ ) and $O B$ (outside the boundary $\Gamma_{h}$ ). The boundary elements $E_{B} \in B$ are further subdivided into sub-elements $E_{\Delta}$ such that $E_{B}=\bigcup_{k=1}^{n} E_{\Delta}$. Sub-elements of an interior boundary element $I B$ are located within the domain $E_{\Delta}=E_{I B}$ whereas the sub-elements of an exterior boundary element $O B$ are located outside $E_{\Delta}=E_{O B}$.

Domain integrals: the interior of the computational domain $\Omega_{h}$, to be considered for the analysis is then defined by the union of the interior elements $(I)$ with the interior boundary sub-elements $(I B)$. Therefore, the integral of a generic function $f$ over a curved computational domain $\Omega_{h}$ is then given by:

$$
\int_{G r_{I \cup B}} \Lambda_{I \cup B} f d \Omega=\int_{I} f d \Omega+\int_{B} \Lambda_{I \cup B} f d \Omega
$$

where

$$
\int_{I} f d \Omega=\sum_{E_{I}} \int_{E_{I}} f d \Omega
$$

and

$$
\begin{aligned}
\int_{B} \Lambda_{I \cup B} f d \Omega & =\sum_{E_{B}} \int_{E_{B}} \Lambda_{I \cup B} f d \Omega \\
& =\sum_{E_{B}} \sum_{E_{\Delta}} \int_{E_{\Delta}} \Lambda_{I \cup B} f d \Omega \\
& =\sum_{E_{B}} \sum_{E_{I B}} \int_{E_{I B}} f d \Omega
\end{aligned}
$$

$\Lambda_{I \cup B}$ is the indicator function [1], taking value 1 if $\left(E_{I}, E_{\Delta}\right) \in \Omega_{h}$ and 0 if $E_{\Delta} \notin \Omega_{h}$.

Boundary integrals: the curved boundaries are approximated by a set of linear segments $E_{\Delta_{\Gamma}}$ in $2 D$ (see Fig. $2 \mathrm{~b}$ ) or triangles in $3 D$ inside a boundary element $E_{B}$. We denote the part of the boundary $\Gamma_{h}$ inside $E_{B}$ by $E_{B_{\Gamma}}$ such that $E_{B_{\Gamma}}=\bigcup_{k=1}^{n} E_{\Delta_{\Gamma}}$. Therefore, the integral of a generic function $f$ over a curved boundary $\Gamma_{h}$ is given by:

$$
\int_{B(\Gamma)} f d \Gamma=\sum_{E_{B_{\Gamma}}} \int_{E_{B_{\Gamma}}} f d \Gamma=\sum_{E_{B_{\Gamma}}} \sum_{E_{\Delta_{\Gamma}}} \int_{E_{\Delta_{\Gamma}}} f d \Gamma
$$

where $B(\Gamma)$ defines the set of boundary elements $B$ that enclose a part or all of the boundary $\Gamma_{h}$. Figure 2c shows an example of boundary integrals over a curved part of boundary $E_{B_{\Gamma}}$ inside a finite element mesh $E_{B}$.

Note that, the integrals over the sub-elements $E_{\Delta}$ and $E_{\Delta_{\Gamma}}$ are based on standard Gauss quadrature. These subelements are only used to generate Gauss points to integrate the weak form and the treatment of Neumann boundary conditions, which differentiates them from finite elements.

\subsection{Numerical examples}

In order to study the influence of the accurate representation of curved domain and the accurate treatment of 


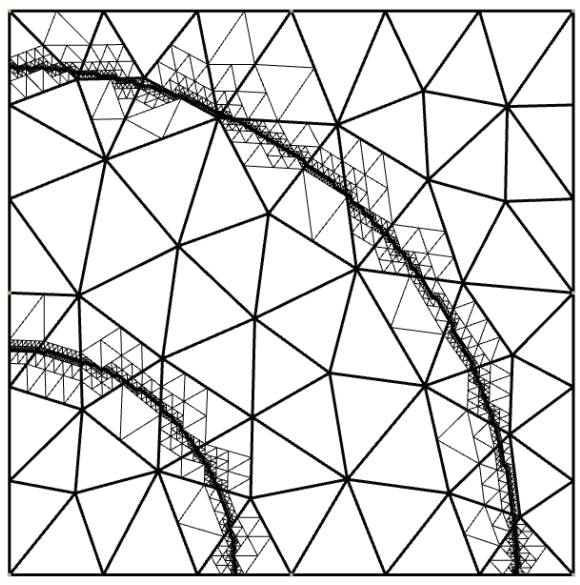

(a)

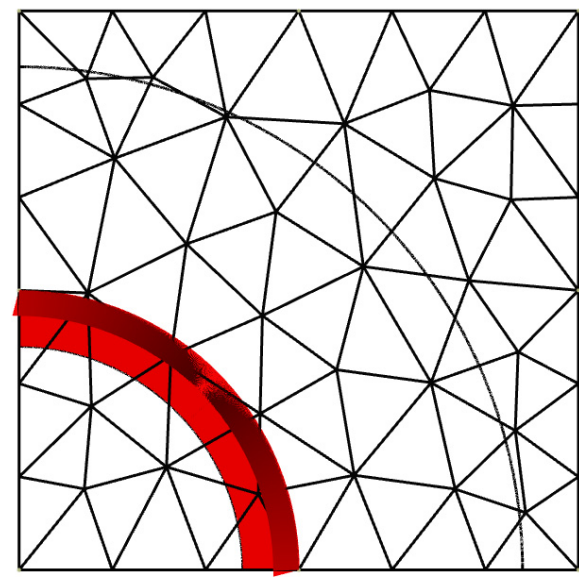

(b)

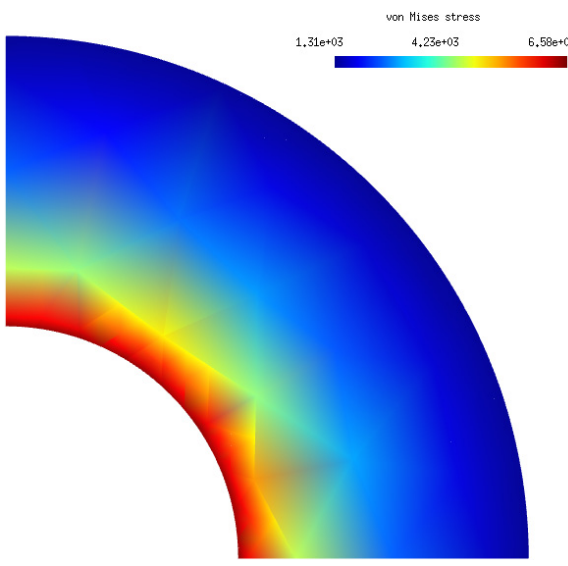

(c)

Fig. 3. A quarter of a thick-wall cylinder under internal pressure. (a) The background coarse mesh and a graded sub-mesh (GSM) of level $(n=10)$. (b) Correct imposition of pressure over the curved internal boundary. (c) Von Mises stress distribution using cubic element.

numerical quadrature on curved boundaries using higherorder XFEM, we analyze the relative error in the energy norm (Eq. (3)) and convergence rates for a test example with known analytical solution. Note that, for a smooth problem, the rate at which the energy error decreases as a uniform mesh is refined is $O\left(h^{p}\right)$, where $h$ is the size of finite elements and $p$ is the polynomial order of the shape functions.

$$
\begin{aligned}
& \mathscr{E}\left(\Omega_{h}\right)= \\
& \left(\frac{\int_{G r_{I \cup B}} \Lambda_{I \cup B} \epsilon\left(u^{h}-u^{e x}\right): \mathbf{C}: \epsilon\left(u^{h}-u^{e x}\right) d \Omega}{\int_{G r_{I \cup B}} \Lambda_{I \cup B} \epsilon\left(u^{e x}\right): \mathbf{C}: \epsilon\left(u^{e x}\right) d \Omega}\right)^{1 / 2}
\end{aligned}
$$

Let us consider the axisymmetric analysis of a thickwall cylinder under internal pressure $p=3000 \mathrm{MPa}$ with Young's modulus $E=10^{6} \mathrm{MPa}$ and Poisson's ratio $\nu=0.3$. In this case, plane stress conditions are assumed, in which analytical solutions are known. Only a quarter of the section was considered. The process to construct the computational domain, the accurate boundary integrals of pressure over the curved internal boundary and the result of analysis are depicted in Figure 3. Figure 3a shows the construction of the computational domain over the background coarse mesh used for analysis and the graded sub-mesh (GSM) used to carefully locate the curved internal and external boundaries.

Different background meshes are considered with different levels of sub-mesh refinement. Convergence studies are carried out using linear, quadratic and cubic elements. The results of the convergence study using XFEM are shown in Figures $4 \mathrm{a}$ and $4 \mathrm{~b}$ respectively for linear/quadratic elements and cubic element. The relative error in the energy norm is plotted as a function of the mesh size (log-log plot). In Figure 4, the rate of convergence $\mathrm{R}$ is also indicated for several levels of sub-mesh refinement $\left(n=1\right.$ to 7 ) inside a boundary element $E_{B}$.
From these results, it is clear that the use of the classical description of boundaries with higher-order finite elements leads to suboptimal convergence rates in the analysis. This is explained by the domination of errors in the boundary description over errors of discretization. By using the proposed approach, it is clear that not only the accuracy, but also the convergence rates are increased. In the cases of quadratic and cubic approximations, the benefit of introducing additional sub-meshes along the curved boundaries is immediately apparent, even for only one refinement inside each boundary element $E_{B}$. For quadratic element, GSM refinement of level $(n=2)$ is sufficient to achieve the theoretical rate of convergence, i.e. $O\left(h^{p=2}\right)$. For cubic element, GSM refinement of level $(n=6)$ is needed to achieve the theoretical rate of convergence, i.e. $O\left(h^{p=3}\right)$.

This methodology shows significant improvement in quality of the solution until the theoretical rate of convergence, i.e. $O\left(h^{p}\right)$, is attained. This means that correct treatment of numerical integration over (i) a curved domain and (ii) on a curved element boundary inside a boundary element $E_{B}$ are achieved with success using a non-conforming mesh.

However, three drawbacks are present in the above approach for practical use:

- For a fixed $p>=2$ polynomial order of the discretization, the computational analysis shows that the level number $n$ used to generate local GSM refinement inside a boundary element $E_{B}$ for achieving the optimal rate of convergence is different in the case of coarse and fine meshes. A constant ratio $h / n$ cannot give the optimal convergence for all background meshes. Supplementary refinement of the GSM level is necessary as the background mesh is refined to provide the optimal convergence. This is because GSM refinement generates linear geometric approximations of the curved boundaries inside a boundary element $E_{B}$. 


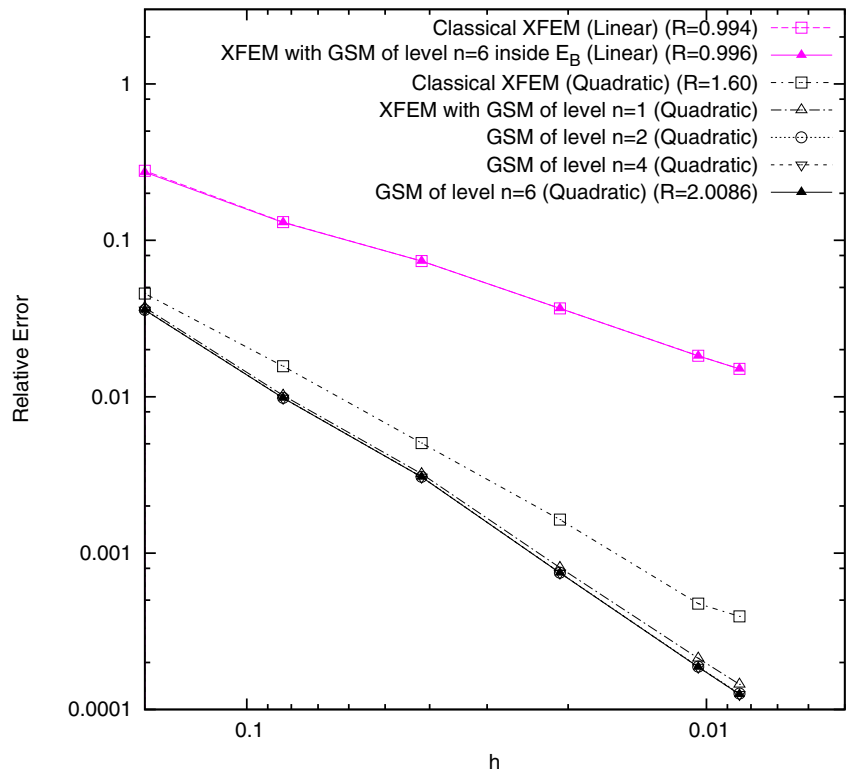

(a)

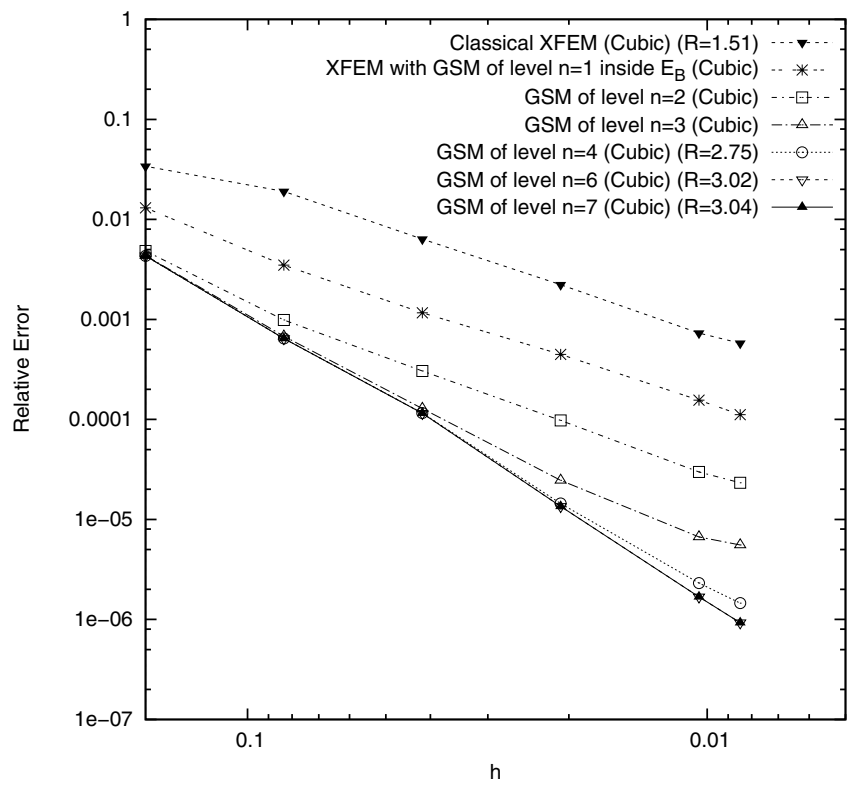

(b)

Fig. 4. Convergence results: (a) linear and quadratic elements, (b) cubic element.

- GSM refinement increases the number of sub-elements which increases the number of integration points for both domain and boundary integrals inside a boundary element.

- An appropriate user-specified level of GSM refinement is necessary to get an optimal solution accuracy for a given background mesh and polynomial order of the discretization.

For the last drawback, it is possible to define a criterion for this particular problem in order to set up the required number of GSM refinement for a given polynomial order of the discretization. However, this approach has been applied on a simple example (an infinite plate with a central hole) which achieves a higher-order rate of convergence. Nevertheless, the required level n of GSM is different from the previous example. Consequently, we cannot establish a simple general criterion, we think that the level number $n$ of GSM depends strongly on local curvature of boundaries, background mesh size and boundary conditions.

Despite these drawbacks, this approach remains an easy and accurate alternative to model geometrical features independently of the finite element mesh size used for analysis (see Fig. 1 for 2D and Fig. 5 for 3D). Also, it guarantees the desired approximation a priori of the original domain geometry by using different levels of the graded sub-mesh (GSM) refinement without changing the background mesh size. Furthermore, computational analysis using higher-order finite element leads, most importantly, to a small size of the resulting matrix to be solved, compared to advanced conforming mesh techniques like h-refinement, for a similar accuracy. For

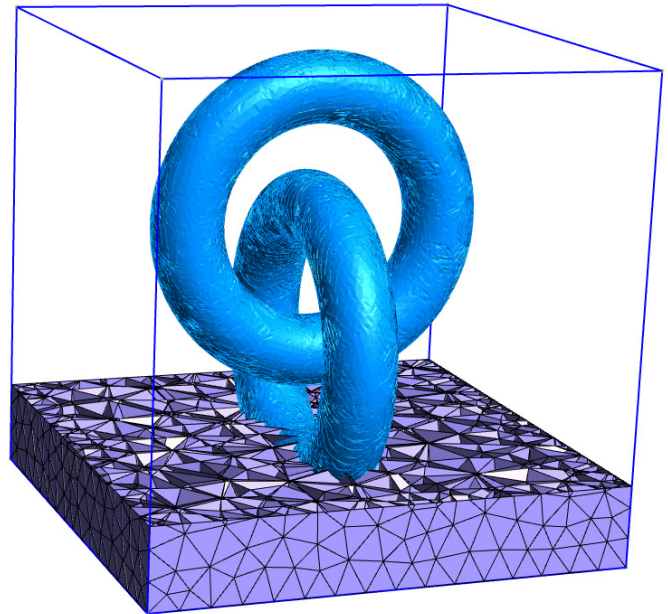

Fig. 5. Modelling a microstructure using a background coarse mesh and GSM refinement independently of the finite element mesh size.

illustration, Figure 6 provides a numerical example of traction using the microstructure of Figure 1.

\section{Conclusions}

Instead of the use of a conforming curved mesh to represent curved boundaries and to perform higher-order finite element analysis, the use of the above framework (non-conforming mesh, parametric functions, graded submesh (GSM) and higher-order XFEM) simplifies mesh generation, achieves the optimal accuracy and higherorder convergence. 


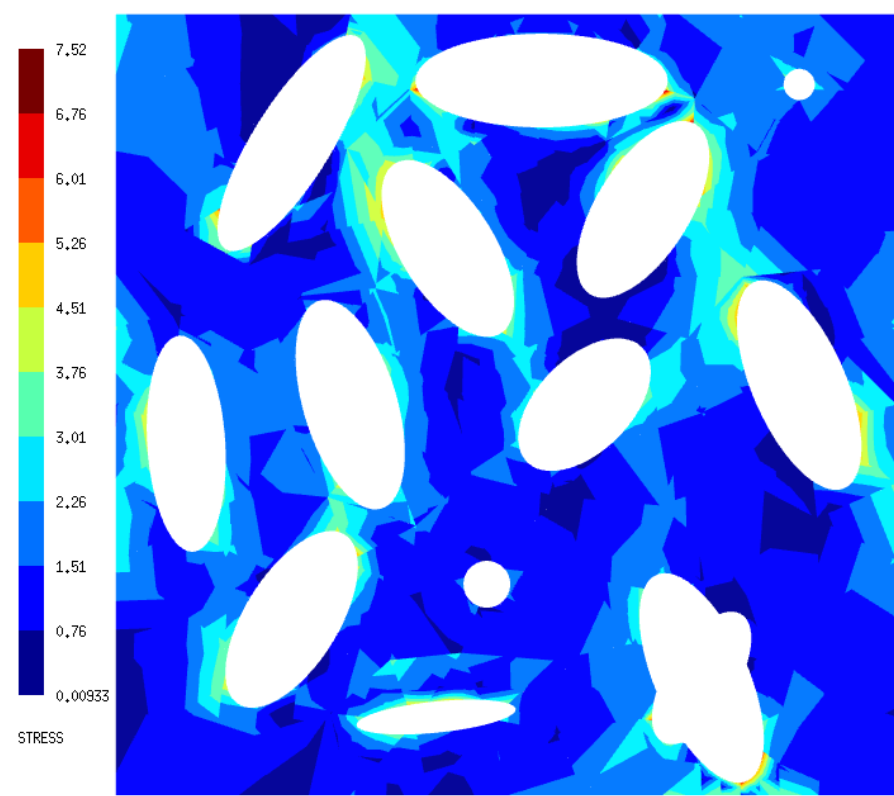

(a)

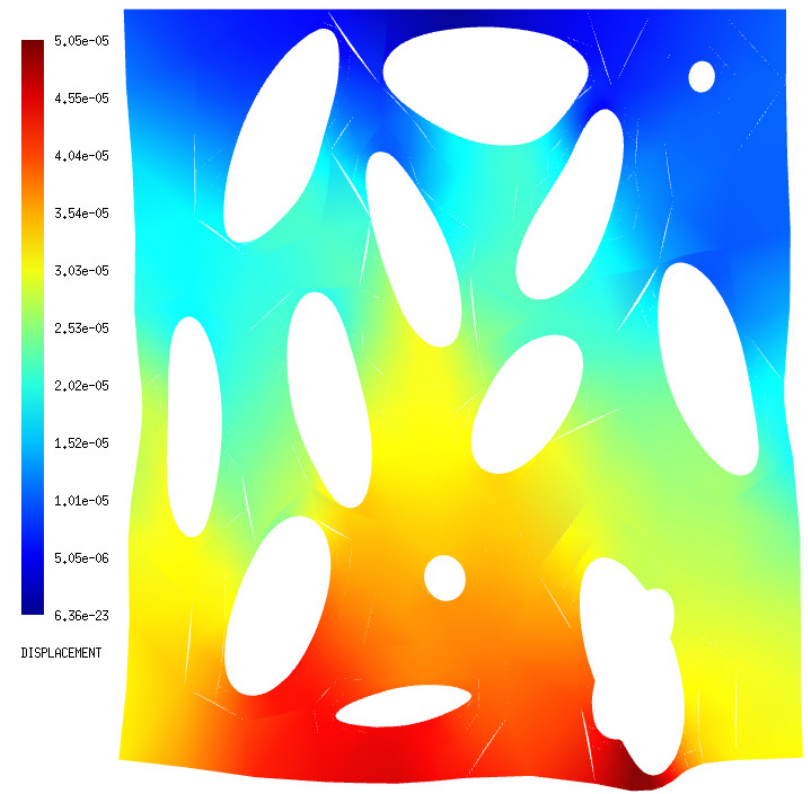

(b)

Fig. 6. Von Mises stress distribution (a) and displacement field (b) using cubic element.

\section{References}

[1] M. Moumnassi, S. Belouettar, E. Béchet, S.P. Bordas, D. Quoirin, M. Potier-Ferry, Finite element analysis on implicitly defined domains: An accurate representation based on arbitrary parametric surfaces, Comput. Methods Appl. Mech. Eng. 200 (2011) 774-796

[2] A. Düster, J. Parvizian, Z. Yang, E. Rank, The finite cell method for three-dimensional problems of solid mechanics, Comput. Methods Appl. Mech. Eng. 197 (2008) 3768-3782

[3] K. Dréau, N. Chevaugeon, N. Moës, Studied x-fem enrichment to handle material interfaces with higher order finite element, Comput. Methods Appl. Mecha. Eng. 199 (2010) 1922-1936

[4] G. Legrain, N. Chevaugeon, K. Dréau, High order x-fem and levelsets for complex microstructures: Uncoupling geometry and approximation, Comput. Methods Appl. Mech. Eng. 241-244 (2012) 172-189

[5] J.P. Pereira, C.A. Duarte, D. Guoy, X. Jiao, hp-generalized fem and crack surface representation for non-planar 3-d cracks, Int. J. Numer. Methods Eng. 77 (2009) 601-633
[6] M. Kästner, S. Müller, J. Goldmann, C. Spieler, J. Brummund, V. Ulbricht, Higher-order extended fem for weak discontinuities-level set representation, quadrature and application to magneto-mechanical problems, Int. J. Numer. Methods Eng. 93 (2013) 1403-1424

[7] E. Nadal, J.J. Ródenas, J. Albelda, M. Tur, J.E. Tarancón, F.J. Fuenmayor, Efficient finite element methodology based on cartesian grids: Application to structural shape optimization, Abstract and Applied Analysis 2013 (2013) 953786

[8] N. Moës, J. Dolbow, T. Belytschko, A finite element method for crack growth without remeshing, Int. J. Numer. Methods Eng. 46 (1999) 131-150

[9] T. Strouboulis, K. Copps, I. Babuška, The generalized finite element method, Comput. Methods Appl. Mech. Eng. 190 (2001) 4081-4193

[10] M. Joulaian, A. Düster, Local enrichment of the finite cell method for problems with material interfaces, Comput. Mech. 52 (2013) 741-762

[11] B.A. Benowitz, H. Waisman, A spline-based enrichment function for arbitrary inclusions in extended finite element method with applications to finite deformations, Int. J. Numer. Methods Eng. 95 (2013) 361-386 